\title{
Electron bubbles in liquid helium: Density functional calculations of infrared absorption spectra
}

\author{
Víctor Grau, Manuel Barranco, Ricardo Mayol, and Martí Pi \\ Departament ECM, Facultat de Física, Universitat de Barcelona, Diagonal 647, 08028 Barcelona, Spain
}

(Received 23 June 2005; published 6 February 2006)

\begin{abstract}
Within density functional theory, we have calculated the energy of the transitions from the ground state to the first two excited states in the electron bubbles in liquid helium at pressures from zero to about the solidification pressure. For ${ }^{4} \mathrm{He}$ at low temperatures, our results are in very good agreement with infrared absorption experiments. Above a temperature of $\sim 2 \mathrm{~K}$, we overestimate the energy of the $1 s-1 p$ transition. We attribute this to the break down of the Franck-Condon principle due to the presence of helium vapor inside the bubble. Our results indicate that the $1 s-2 p$ transition energies are sensitive not only to the size of the electron bubble, but also to its surface thickness. We also present results for the infrared transitions in the case of liquid ${ }^{3} \mathrm{He}$, for which we lack experimental data.
\end{abstract}

DOI: 10.1103/PhysRevB.73.064502

PACS number(s): 67.40.Yv, 67.55.Lf, 68.03.Cd, 47.55.D-

\section{INTRODUCTION}

Excess electrons in liquid helium are known to form electron bubbles. This is so because of the strong repulsion between the helium atoms, which are very weakly polarizable, and the intruder electrons. Experimental and theoretical spectroscopic studies on electron bubbles have been carried out for many years since the pioneering work of Northby and Sanders $^{1}$ and of Dexter and co-workers.,3 Only some 20 years later, Grimes and Adams were able to carried out a detailed analysis of the infrared absorption spectrum of the electron bubble in liquid ${ }^{4} \mathrm{He}$. In a first work, ${ }^{4}$ they used a photoconductive mechanism to detect the transition which operated only over a limited region of the pressuretemperature $(P-T)$ plane because it appeared to be associated with trapping of bubbles on vortices in the superfluid. Later on, they improved their experimental apparatus and observed the electronic transitions in direct infrared absorption. ${ }^{5}$ This allowed them to study the spectrum of electron bubbles in a wider region of the $P-T$ plane from $P=0$ to the solidification pressure, and up to temperatures above $4 \mathrm{~K}$ instead of the maximum $T \sim 1.6 \mathrm{~K}$ reached in photocurrent experiments.

Recent experiments on cavitation in liquid helium have renewed interest in the study of single-electron bubbles. ${ }^{6-8}$ Multielectron bubbles have also prompted some theoretical activity; ${ }^{9,10}$ while the stability of multielectron bubbles is not yet clarified, ${ }^{10,11}$ single-electron bubbles are stable entities for pressures above saturation pressure. Electron bubbles in helium droplets are metastable objects whose lifetime has been measured for both helium isotopes. ${ }^{12}$

The simplest model to address electron bubbles in liquid helium supposes that they are confined in a spherical well potential of radius $R$. The total energy of the electron-helium $(e-\mathrm{He})$ system is then written as a function of $R$,

$$
U(R)=E_{e}+4 \pi R^{2} \sigma+\frac{4}{3} \pi R^{3} P,
$$

where $E_{e}$ is the ground-state electronic energy, $P$ is the pressure applied to the system, and $\sigma$ is the surface tension of the liquid. For an infinite spherical well potential, $E_{e}$ $=\pi^{2} \hbar^{2} /\left(2 m_{e} R^{2}\right)$. This model is able to qualitatively repro- duce the experimental infrared absorption energies, and is simple enough to allow to calculate shape fluctuations of electron bubbles and line shapes. ${ }^{13}$ It can be refined ${ }^{2-5}$ by taking into account the finite depth of the well $V_{0}$, which is about $1 \mathrm{eV} .{ }^{14}$ Once $U(R)$ has been minimized with respect to $R$ and the radius of the equilibrium bubble $R_{e q}$ has been determined, it is easy to obtain the energies of the $(n, l)$ excited states and to compute the transition energies to the ground state if the Franck-Condon principle holds, i.e., if the absorption of a photon by the electron bubble is a process much faster than the time needed for the helium bubble to adapt itself to the electron wave function in the excited state.

Another key ingredient entering Eq. (1) is the surface tension $\sigma$, which for ${ }^{4} \mathrm{He}$ at zero $T$ and $P$ is about 0.274 $\mathrm{K} \AA^{-2}{ }^{15}$ This yields $R_{e q}=18.9 \AA$. The surface tension is only known along the liquid-vapor coexistence line. Consequently, to use Eq. (1) one has to rely on model calculations of $\sigma(P, T)$. The situation has been discussed in detail by Grimes and Adams, who concluded that in order to perfectly fit their experimental results with Eq. (1), one has to take the depth of the well $V_{0} P$ dependent and a surface tension $\sigma$ independent of $P$, which seems difficult to justify. For instance, early calculations ${ }^{3,17}$ indicated that the surface tension $\sigma$ nearly doubles from $P=0$ to 25 atm. For the sake of completeness, we present in the Appendix a calculation of $\sigma(P)$ that also yields this increase, although we will not make substantial use of these results in our study. It is worthwhile to stress that when both $V_{0}$ and $\sigma$ were made $P$ dependent following the expected pressure dependences, the agreement between theory ${ }^{3}$ and experiment was only qualitative (see Fig. 2 of Ref. 4). For this reason, these authors concluded that more sophisticated calculations of the properties of electron bubbles were called for, incorporating the fact that the bubble density profile is not abrupt but the bubbles have a finite surface thickness.

In this work we present a theoretical description of infrared absorption $1 s-1 p$ and $1 s-2 p$ excitation energies of electron bubbles in liquid helium. It belongs to the class of the more sophisticated calculations suggested by Grimes and Adams, since it is not based on ad hoc assumptions, but on the use of a finite-temperature density functional (DF) ap- 
proach in conjunction with a realistic electron-helium effective potential. DF methods have become increasingly popular in recent years as useful computational tools to study the properties of classical and quantum inhomogeneous fluids, ${ }^{18}$ especially for large systems for which these methods provide a good compromise between accuracy and computational cost, yielding results in agreement with experiment or with more microscopic approaches. In the frame of DF theory, the properties of an electron bubble approaching the surface of liquid ${ }^{4} \mathrm{He}$ have been studied by Ancilotto and Toigo ${ }^{19}$ using the so-called Orsay-Paris (OP) zero-temperature finite-range DF (Ref. 20) and the pseudopotential proposed in Ref. 21 as $e$-He interaction. This is another key ingredient of the calculation, and an accurate description of the infrared absorption can only be achieved using an accurate $e$-He interaction. We want to mention that DF theory has been successfully applied to dynamical problems in bulk liquid helium and helium droplets ${ }^{22-24}$ using a generalization of the OP density functional called the Orsay-Trento (OT) functional. ${ }^{25}$ Having no ad hoc parameters to be adjusted in the calculation to describe the infrared absorption energies, we will show that the simplest form of a DF for liquid helium, namely, a zerorange one, is not able to reproduce the experimental data. As we are going to show, one must resort to more sophisticated forms, such as the mentioned OP and OT finite-range functionals. This finding is additional supporting evidence of the reliability of finite-range functionals.

Density functional theory has also proven to be the most successful approach in addressing cavitation in liquid helium so far. $^{26-28}$ It incorporates in a self-consistent way the equation of state of the bulk liquid and the surface tension of the liquid-gas interface as a function of temperature. It allows for a flexible description of the electron bubble, incorporating surface thickness effects. Within DF theory one avoids the use of macroscopic concepts such as surface tension and pressure at a nanoscopic scale; however, it is a continuous, not an atomic description of the system. Used in conjunction with a Hartree-type $e$-He potential, we have recently shown that this approach quantitatively reproduces the existing experimental data on cavitation of electron bubbles in liquid helium below saturation pressure. ${ }^{29}$ Consequently, it is a tested framework to address other properties of electron bubbles.

This paper is organized as follows. In Sec. II we briefly present the density functional plus Hartree electron effective potential approach we have employed, as well as some numerical details. The structure of the electron bubble (radius and surface thickness) and infrared absorption energies are discussed in Sec. III, and a summary is presented in Sec. IV. Finally, we present in the Appendix a model calculation of the surface tension as a function of pressure that we have used to make some crude estimates of the electron bubble radius.

\section{DENSITY FUNCTIONAL APPROACH AND ELECTRON-HELIUM INTERACTION}

As in Ref. 29, our starting point is the finite-temperature zero-range DF of Ref. 30 that reproduces thermal properties of liquid ${ }^{4} \mathrm{He}$ such as the experimental isotherms and the ${ }^{4} \mathrm{He}$ liquid-gas coexistence line up to $T=4.5 \mathrm{~K}$, and the $T$ dependence of the surface tension of the liquid free surface. We have taken the Hartree-type $e$-He effective potential derived by Cheng et al. ${ }^{31}$ (see also Ref. 32) as $e$-He interaction. This allows us to write the free energy of the system as a functional of the ${ }^{4} \mathrm{He}$ particle density $\rho$, the excess electron wave function $\Psi$, and $T$,

$$
\begin{aligned}
F[\rho, \Psi, T]= & \int d \vec{r} f(\rho, T)+\frac{\hbar^{2}}{2 m_{e}} \int d \vec{r}|\nabla \Psi(\vec{r})|^{2} \\
& +\int d \vec{r}|\Psi(\vec{r})|^{2} V(\rho),
\end{aligned}
$$

where $f(\rho, T)$ is the ${ }^{4} \mathrm{He}$ free-energy density per unit volume written as

$$
f(\rho, T)=f_{v o l}(\rho, T)+\beta \frac{(\nabla \rho)^{2}}{\rho}+\xi(\nabla \rho)^{2} .
$$

In this expression, $f_{v o l}(\rho, T)$ consists of the free-energy density of the Bose gas, plus phenomenological density- and temperature-dependent terms written as

$$
f_{\text {int }}(\rho, T)=\frac{1}{2} b(T) \rho^{2}+\frac{1}{2} c(T) \rho^{2+\gamma}
$$

which takes into account the effective interaction of helium atoms in the bulk liquid. The parameters of these terms and those of the density gradient terms in Eq. (3) have been adjusted so as to reproduce physical quantities like the equation of state of the bulk liquid and the surface tension of the liquid free surface.

The $e-\mathrm{He}$ interaction $V(\rho)$ is written as a function of the local helium density ${ }^{31}$

$$
V(\rho)=\frac{\hbar^{2} k_{0}^{2}}{2 m_{e}}+\frac{2 \pi \hbar^{2}}{m_{e}} \rho a_{\alpha}-2 \pi \alpha e^{2}\left(\frac{4 \pi}{3}\right)^{1 / 3} \rho^{4 / 3},
$$

where $\alpha=0.208 \AA^{3}$ is the static polarizability of a ${ }^{4} \mathrm{He}$ atom, and $k_{0}$ is determined from the helium local Wigner-Seitz radius $r_{s}=(3 / 4 \pi \rho)^{1 / 3}$ by solving the trascendental equation

$$
\tan \left[k_{0}\left(r_{s}-a_{c}\right)\right]=k_{0} r_{s},
$$

with $a_{c}$ and $a_{\alpha}$ being the scattering lengths arising from a hard-core and from a polarization potential. We have taken ${ }^{31}$ $a_{\alpha}=-0.06 \AA$ and $a_{c}=0.68 \AA$.

For given $P$ and $T$ values we have solved Euler-Lagrange equations which result from the variation of the constrained grand potential density $\widetilde{\omega}(\rho, \Psi, T)=\omega(\rho, \Psi, T)-\varepsilon|\Psi|^{2}$, where

$$
\omega(\rho, \Psi, T)=f(\rho, T)+\frac{\hbar^{2}}{2 m_{e}}|\nabla \Psi|^{2}+|\Psi|^{2} V(\rho)-\mu \rho .
$$

It yields

$$
\frac{\delta f}{\delta \rho}+|\Psi|^{2} \frac{\partial V}{\partial \rho}=\mu
$$




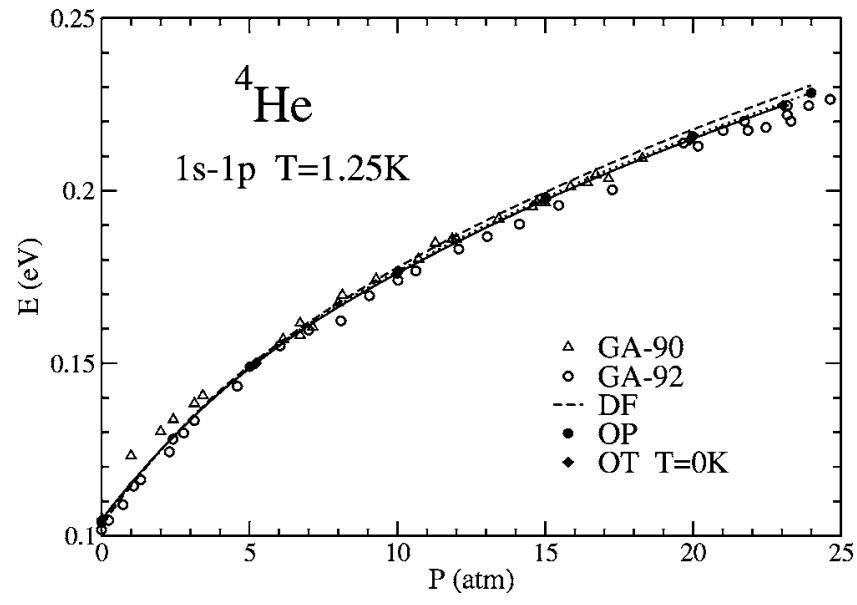

FIG. $1 .{ }^{4} \mathrm{He} 1 s-1 p$ transition energies (eV) as a function of $P$ (atm) for $T=1.25 \mathrm{~K}$. The open triangles are the observed points from the electron bubble photocurrent (Ref. 4), and the open circles correspond to direct infrared absorption measurements (Ref. 5). The dashed line represents the results obtained using the zero-range DF discussed in Sec. II. Filled circles connected with a dotted line are the results obtained using the OP finite-range DF at $T=1.25 \mathrm{~K}$, and filled diamonds connected with a solid line are results obtained using the zero-temperature OT finite-range DF.

$$
-\frac{\hbar^{2}}{2 m_{e}} \Delta \Psi+V(\rho) \Psi=\varepsilon \Psi,
$$

where $\varepsilon$ is the lowest eigenvalue of the Schrödinger equation obeyed by the electron. These equations are solved assuming spherical symmetry, imposing for $\rho$ that $\rho^{\prime}(0)=0$ and $\rho(r$ $\rightarrow \infty)=\rho_{b}$, where $\rho_{b}$ is the density of the bulk liquid, and that the electron is in the $1 s$ state. Fixing $\rho_{b}$ and $T$ amounts to fixing $P$ and $T$, since the pressure can be obtained from the bulk equation of state $P=-f_{v o l}\left(\rho_{b}, T\right)+\mu \rho_{b}$, and the ${ }^{4} \mathrm{He}$ chemical $\mu=\partial f_{v o l}(\rho, T) /\left.\partial \rho\right|_{T}$ is known in advance. We have used a multidimensional Newton-Raphson method ${ }^{33}$ for solving Eqs. (8) and (9) after having discretized them using 13-point formulas for the $r$ derivatives. A fine mesh of step $\Delta r=0.1 \AA$ has been employed, and the equations have been integrated up to $R_{\infty}=150 \AA$ to make sure that the asymptotic bulk liquid has been reached. After obtaining the equilibrium configuration ( $1 s$ state), the spectrum of the electron bubble $\varepsilon_{n l}$ is calculated from Eq. (9) keeping frozen the helium density (Franck-Condon principle).

\section{RESULTS}

\section{A. Liquid ${ }^{4} \mathrm{He}$}

Figure 1 shows the $1 s-1 p$ transition energies $(\mathrm{eV})$ as a function of $P$ (atm) for $T=1.25 \mathrm{~K}$. It can be seen that the agreement between theory and experiment is good from $P$ $=0$ to the solidification pressure, and consequently, the physical process seems well understood. However, a minor discrepancy appears at high pressures, and especially in the description of the $1 s-2 p$ transition energies, as can be seen in Fig. 2. This is not surprising, since one would expect that the $2 p$ state is more sensitive to fine details of the bubble struc-

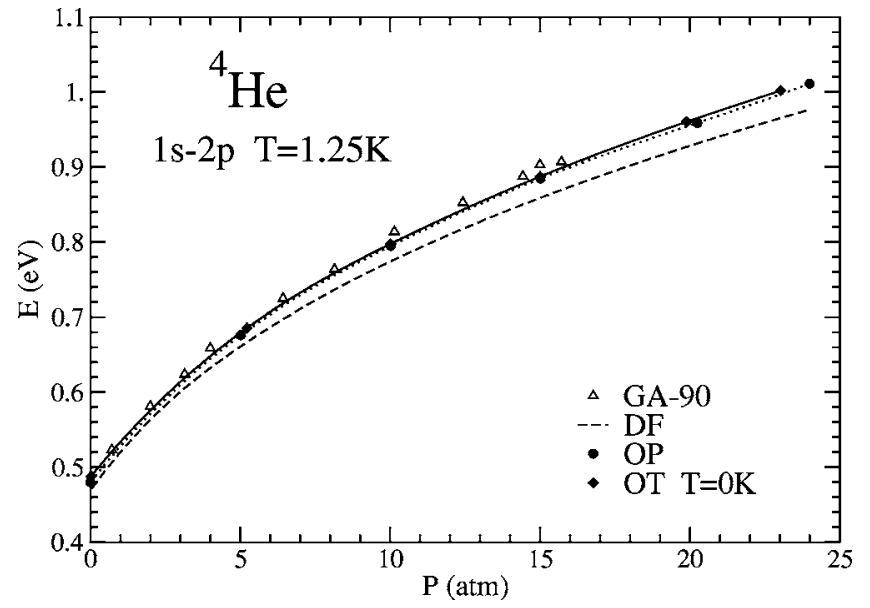

FIG. 2. ${ }^{4} \mathrm{He} 1 s-2 p$ transition energies (eV) as a function of $P$ (atm) for $T=1.25 \mathrm{~K}$. The open triangles are the observed points from the electron bubble photocurrent (Ref. 4). The dashed line represents the results obtained using the zero-range DF discussed in Sec. II. Filled circles connected with a dotted line are the results obtained using the OP finite-range DF at $T=1.25 \mathrm{~K}$, and filled diamonds connected with a solid line are results obtained using the zero-temperature OT finite-range DF.

ture, in particular to its thickness, because the $2 p$ wave function penetrates deeper into the liquid.

We have improved the method of Sec. II to achieve a better agreement with experiment. The improvement is based on the observation that zero-range DF's like the one described in Sec. II are fitted to reproduce the experimental surface tension of liquid helium at zero temperature, ${ }^{30,34}$ and then the $T$ dependence of $\sigma,{ }^{30}$ and the thickness $t$ of the free surface-defined as the difference between the distances at which the density equals $0.1 \rho_{b}$ and $0.9 \rho_{b}$, where $\rho_{b}$ is the bulk density at the given $(P, T)$ - come out as predictions of the formalism. It turns out that at $T=0$, zero-range DF's overestimate $t$ by about $1 \AA$. Indeed, recent measurement of the ${ }^{4} \mathrm{He}$ surface thickness yield values around $6 \AA$, 35,36 whereas the value predicted in Refs. 30 and 34 is about $7 \AA$. In the case of ${ }^{3} \mathrm{He}$, the experimental value is about $7.5 \AA,{ }^{37}$ whereas the prediction using zero-range DF's is about 8.5-9 $\AA .{ }^{34,38}$ We recall that the surface thickness of liquid helium is a quantity rather difficult to determine experimental and theoretically, ${ }^{39}$ and the dispersion of the values assigned to it is large. ${ }^{34-41}$ Only recently have the mentioned values $\left(\sim 6-6.5 \AA\right.$ for ${ }^{4} \mathrm{He}$ and $\sim 7.5 \AA$ for $\left.{ }^{3} \mathrm{He}\right)$ emerged as likely accurate determinations of the surface thickness of the liquid helium free surface.

In the 1990s, a new class of DF's appeared that retains some of the simplicity of the original zero-range DF's, incorporating finite-range effects that are absolutely necessary to address a wide class of physical phenomena, like elementary excitations in bulk liquids and large inhomogeneities caused by the presence of impurities in bulk liquid and droplets, and also by substrates. Two such DF's are the OP and OT functionals for ${ }^{4} \mathrm{He}$ already noted. ${ }^{20,25}$ It is remarkable that they reproduce the experimental surface tension at $T=0$ without having imposed it in their construction, and also the surface thickness. In particular, the OP functional yields $\sigma=0.277$ 


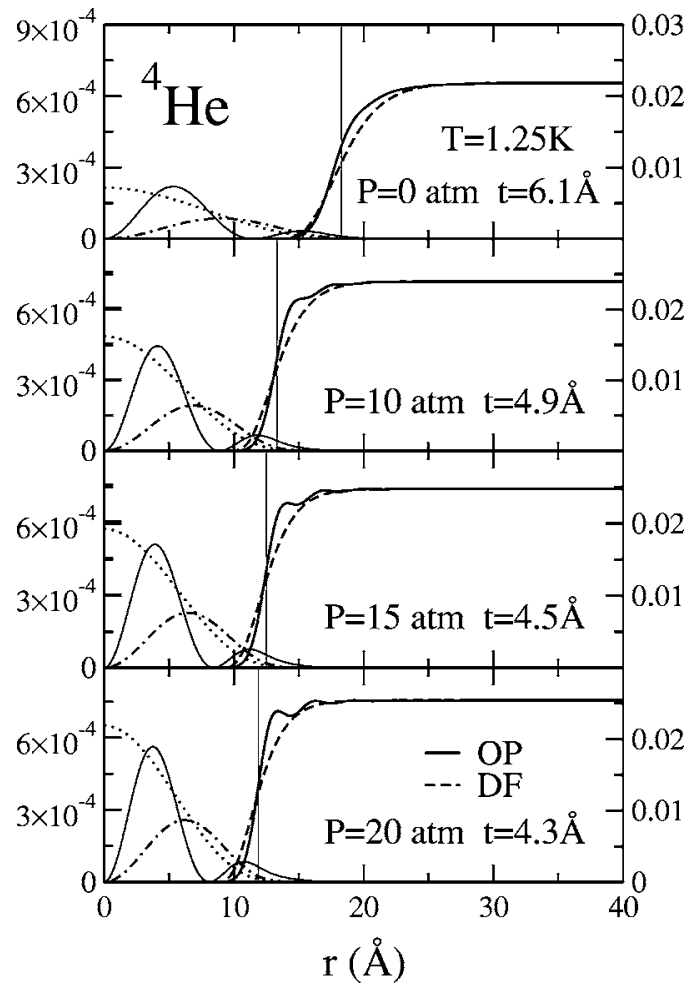

FIG. 3. Electron bubble density profiles in $\AA^{-3}$ (right scale) and excess electron squared wave functions $|\Psi|^{2}$ in $\AA^{-3}$ (left scale), as a function of the radial distance $r(\AA)$ for $T=1.25 \mathrm{~K}$ and different pressures. The dashed lines correspond to the zero-range DF, and the solid lines to the OP functional. The vertical thin lines indicate the equilibrium radius $R_{e q}$ yielded by the simple electron bubble model Eq. (1) using the values of $\sigma(P)$ obtained in the Appendix. The value of the surface thickness $t$ is also given for the zero-range DF. The electron squared wave functions $|\Psi|^{2}$ correspond to the OP calculation; dotted line, $1 s$ state; dot-dashed line, $1 p$ state; thin solid line, $2 p$ state.

$\mathrm{K} \AA^{-2}, t=5.8 \AA$, and the OT functional yields $\sigma=0.272$ $\mathrm{K} \AA^{-2}, t=6 \AA^{20,25}$ A recent diffusion Monte Carlo calculation yields $\sigma=0.281(3) \mathrm{K}^{-2}, t=6.3(4) \AA^{41}$

It is then quite natural to ask oneself if the remaining disagreement between theory and experiment shown in Fig. 1 and especially in Fig. 2 might arise from a worse description of the electron bubble surface in the case of zero-range DF's. Indeed, the error in the infrared energies has been estimated $^{4}$ to be $\sim 1 \%$, smaller than the difference between experiment and zero-range DF calculations. To answer this question, we have repeated the calculations using the zerotemperature OT functional-thermal effects on the excitation energies are expected to be small for $T=1.25 \mathrm{~K}$, and this is what we have found (see below) — and also using a straightforward generalization at finite $T$ of the OP functional, which is possible because of the similarities between the OP and the zero-range DF of Sec. II. ${ }^{42}$ It is worth knowing that a generalization of the OT density functional at finite temperatures is also available. ${ }^{43}$

Density profiles of electron bubbles at different pressures and $T=1.25 \mathrm{~K}$, obtained with the $\mathrm{OP}$ and the zero-range functional, are shown in Fig. 3, and in Fig. 4 for the OT

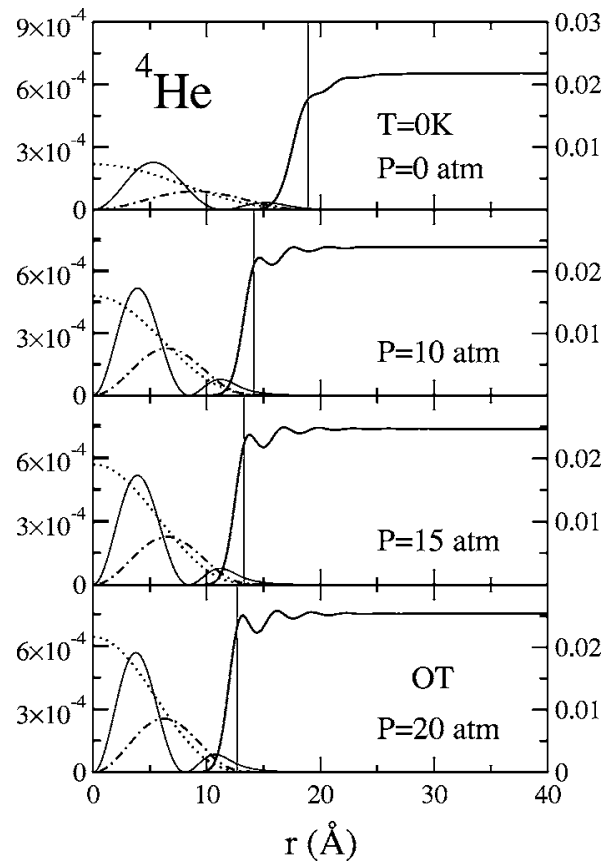

FIG. 4. Electron bubble density profiles in $\AA^{-3}$ (right scale) and excess electron squared wave functions $|\Psi|^{2}$ in $\AA^{-3}$ (left scale), as a function of the radial distance $r(\AA)$ for $T=0 \mathrm{~K}$ and different pressures, obtained using the OT functional. The electron squared wave functions $|\Psi|^{2}$ are represented as in Fig. 3, i.e., dotted line, $1 s$ state; dot-dashed line, $1 p$ state; thin solid line, $2 p$ state. The vertical thin lines indicate the equilibrium radius $R_{e q}$ yielded by the simple electron bubble model Eq. (1) using the values of $\sigma(P)$ obtained in the Appendix.

functional at $T=0 \mathrm{~K}$. The excess electron squared wave functions $|\Psi|^{2}$ corresponding to the $1 s, 1 p$, and $2 p$ states are also represented. For the zero-range DF we also give the value of the surface thickness $t$, which goes from $6.1 \AA$ at $P=0 \mathrm{~atm}$ to $4.3 \AA$ at $P=20 \mathrm{~atm}$. In the case of the OP, and especially of the OT functional, it is difficult to define the thickness, and even the bubble radius, because of the density oscillations in the surface region (see also Ref. 22). For reference, we indicate that the surface thickness of the electron bubble at $T=1.25 \mathrm{~K}$ and $P=0 \mathrm{~atm}$ obtained with the OP functional is $5 \AA$, one $\AA$ smaller than the value yielded by the zero-range $\mathrm{DF}$.

Figures 1 and 2 also show the results obtained with OP and OT functionals. It can be seen that in this case, the agreement between theory and experiment is excellent. ${ }^{44}$ It is worthwhile to mention that Eloranta and Apkarian ${ }^{22}$ have also obtained the absorption energies up to $P \sim 12$ atm using the OT functional and the pseudopotential of Jortner et al. ${ }^{45}$ as $e$-He interaction. Their results for the $1 s-2 p$ transition energies are not in as good agreement with experiment as ours, likely because of the different $e$-He interaction we have used. In addition, the relevance of a correct description of the surface thickness to reproduce the experimental data has been overlooked in their work, which is mainly about the dynamics of electron bubble expansion in liquid ${ }^{4} \mathrm{He}$.

Figure 5 shows the evolution of the radius of the electron bubble with pressure determined by several authors using 


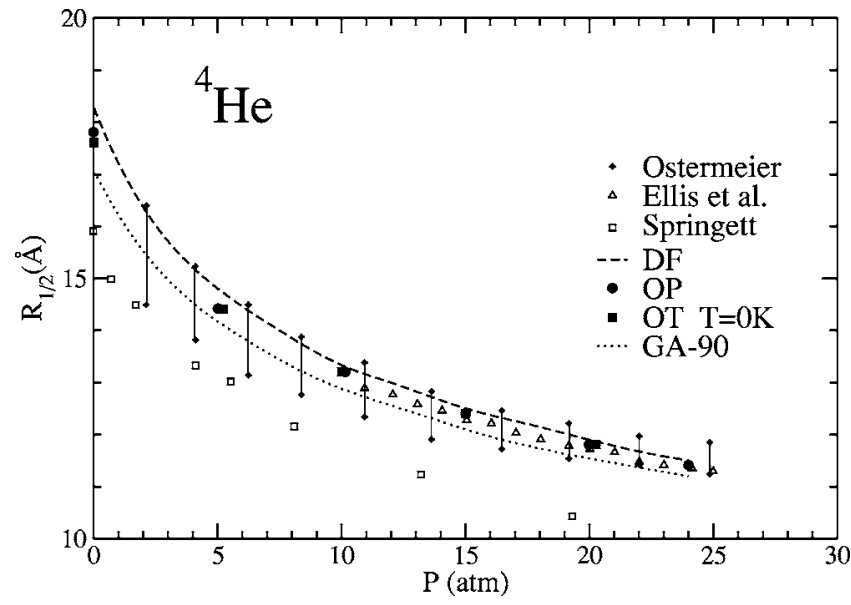

FIG. 5. Radius of the electron bubble as a function of pressure. Dashed line, zero-range DF results; filled circles (squares), OP (OT) results at $T=1.25(0) \mathrm{K}$; dotted line, Ref. 4 results. Open squares, data with error bars, and open triangles are from Refs. 46-48, respectively.

different methods, ${ }^{4,46-48}$ and the value we have obtained using the zero-range and the OP and OT functionals. For a given pressure, we have defined the radius of the bubble as the radial distance at which the helium density reaches the corresponding bulk value divided by 2 . It can be seen that our results are compatible with the more recent experimental determinations, even those obtained with the zero-range DF, which overestimate the radius of the electron bubble by $\sim 0.5 \AA$.

We present in Fig. 6 the variation of the $1 s-1 p$ excitation energy with $T$ at $P=2.9$ atm. Open circles are the experimental data of Grimes and Adams, ${ }^{5}$ and the dashed line is the result obtained by these authors using their spherical-squarewell (SSW) model with the $T$ dependence of the surface tension as at saturation vapor pressure. ${ }^{16}$ Our results, displayed as filled circles, have been obtained using the finite-

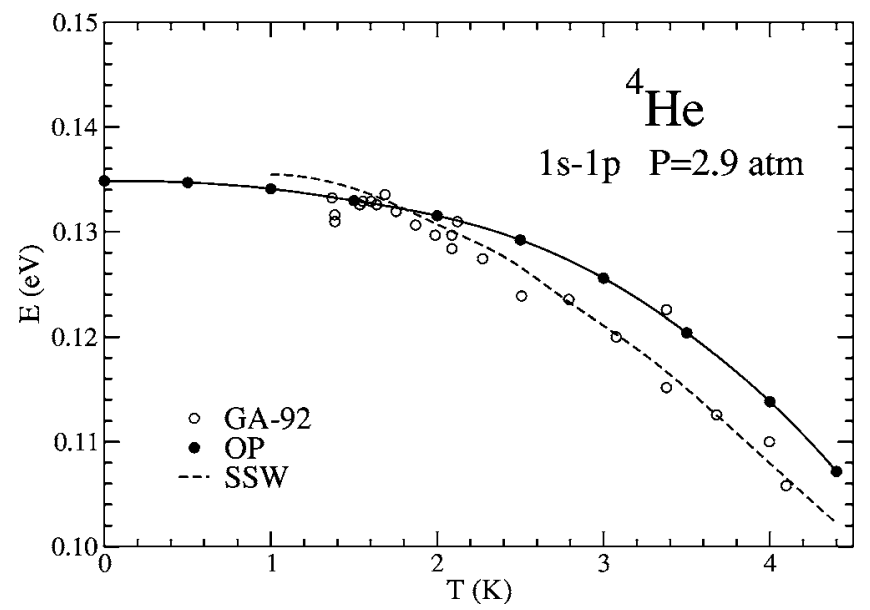

FIG. 6. Variation of the $1 s-1 p$ excitation energy with $T$ at $P$ $=2.9 \mathrm{~atm}$. Open circles are the experimental data of Ref. 5. Dashed line, SSW model results (Ref. 5). Filled circles, finite-temperature OP functional results. The solid line has been drawn to guide the eye.
TABLE I. Transition energies, oscillator strengths, and total cross sections for the first two dipole excitations of electron bubbles in ${ }^{4} \mathrm{He}$ calculated with the OT functional at $T=0$ and $P=0 \mathrm{~atm}$.

\begin{tabular}{cccc}
\hline \hline Transition & $\begin{array}{c}\text { Transition } \\
\text { energy }(\mathrm{eV})\end{array}$ & $\begin{array}{c}\text { Oscillator } \\
\text { strength }\end{array}$ & $\begin{array}{c}\text { Total cross } \\
\text { section }\left(\mathrm{eV} \mathrm{cm}{ }^{2}\right)\end{array}$ \\
\hline $1 s-1 p$ & 0.105 & 0.971 & $1.07 \times 10^{-16}$ \\
$1 s-2 p$ & 0.488 & 0.0250 & $2.75 \times 10^{-18}$ \\
\hline
\end{tabular}

temperature OP functional. It can be seen that above $T$ $=2 \mathrm{~K}$ we overestimate the excitation energies. In spite of the good agreement between experiment and the SSW model, we believe it is coincidental, and that the failure of our method to describe the experiment above $T=2 \mathrm{~K}$ is the physical signature of the breaking down of the FranckCondon principle at high $T$. Indeed, above $T \sim 2 \mathrm{~K}$, it is no longer valid to assume that the electron bubble is void of $\mathrm{He}$ atoms. It has been shown ${ }^{6,29}$ that the bubble is progressively filled with helium vapor that must be "dragged" by the electron in the course of its dipole excitation. This dynamical effect has to be taken into account if one wants to have a quantitative agreement with experiment above $T \sim 2 \mathrm{~K}$. Although it is in principle possible to address this problem within time-dependent density functional theory ${ }^{23}$ using the finite-temperature OP functional or that of Ref. 43, this is not a trivial issue and it is beyond the scope of our work.

Finally, we have also calculated the cross section of the transition from the $1 s$ to the $1 p$ and $2 p$ excited states. For photons of frequency $w$ polarized along the $z$ axis, the cross section is given by

$$
\sigma(w)=\frac{4 \pi^{2} e^{2}}{c} w|\langle n p|z| 1 s\rangle|^{2} \delta\left(E_{n p}-E_{1 s}-\hbar w\right) .
$$

We collect in Table I the excitation energies $(\mathrm{eV})$, oscillator strengths, and total cross sections $\left(\mathrm{eV} \mathrm{cm}^{2}\right)$ to the first two $p$ states obtained with the OT functional at $T=0, P$ $=0 \mathrm{~atm}$. Our results are in full agreement with those of Ref. 2.

\section{B. Liquid ${ }^{3} \mathrm{He}$}

We have also studied the infrared absorption spectrum for electron bubbles in liquid ${ }^{3} \mathrm{He}$. The application of DF theory to describe electron bubble explosions in ${ }^{3} \mathrm{He}$ proceeds as shown in Sec. II. We have used the zero-range DF proposed in Ref. 38 to describe the inhomogeneous liquid, and also a finite-range (FR) DF, obtained from the zero-range one following the procedure indicated in Ref. 20 for the ${ }^{4} \mathrm{He}$ case. The $e$-He interaction is given again by Eq. (5) with the parameters corresponding to ${ }^{3} \mathrm{He}$, namely, $\alpha=0.206 \AA^{3}$, and the same values for $a_{\alpha}$ and $a_{c}$.

We represent in Fig. 7 the infrared absorption energies for the $1 s-1 p$ and $1 s-2 p$ transitions. It can be seen that the results are qualitatively similar to those found for ${ }^{4} \mathrm{He}$, i.e., a fair insensitivity of the $1 s-1 p$ transition energy to the detailed structure of the bubble surface, and an underestimation of the $1 s-2 p$ transition energy by the zero-range DF. The half- 

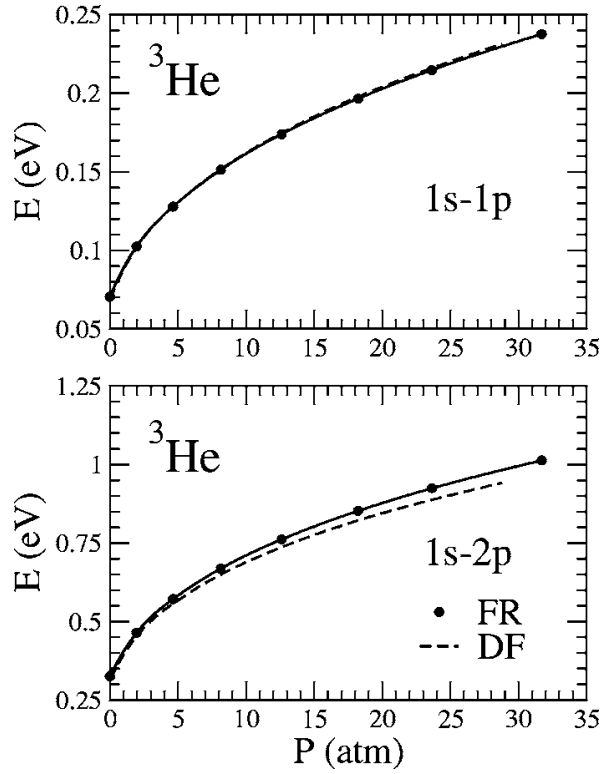

FIG. 7. ${ }^{3} \mathrm{He} 1 s-1 p$ and $1 s-2 p$ transition energies (eV) as a function of $P(\mathrm{~atm})$ for $T=0 \mathrm{~K}$. The dashed line is the result obtained using the zero-range DF of Ref. 38. Filled dots connected with a solid line are the results obtained using the zero-temperature finiterange DF.

density radius of the electron bubble $R_{1 / 2}$ goes from $22.5 \AA$ at $P=0$ to $11.8 \AA$ at $P=22.3 \mathrm{~atm}$.

The smaller excitation energies in the case of ${ }^{3} \mathrm{He}$ are due to the smaller surface tension for this isotope, $\sigma$ $=0.113 \mathrm{~K}^{-2}$, 49 which causes electron bubble radii to be larger for ${ }^{3} \mathrm{He}$ than for ${ }^{4} \mathrm{He}$ (e.g., $R_{e q}=23.5 \AA$ at $P=0$ instead of $18.9 \AA$ ), yielding smaller excitation energies, as shown for instance by the simple model of Eq. (1). In particular, at $P$ $=0$ the model gives for the ratio of the $1 s-1 p$ excitation energies the value $\left(\sigma_{3} / \sigma_{4}\right)^{1 / 2}$, in good agreement with the results obtained within the DF approach (see the tables).

Finally, we collect in Table II the excitation energies $(\mathrm{eV})$, oscillator strengths, and total cross sections $\left(\mathrm{eV} \mathrm{cm}^{2}\right)$ to the first two $p$ states obtained with the FR functional at $T=0$, $P=0$ atm. It can be seen that the oscillator strengths and cross sections are sensibly the same for both isotopes.

\section{SUMMARY}

We have demonstrated the suitability of the density functional approach to quantitatively address electron bubbles in liquid He. On the one hand, we have shown that the DF

TABLE II. Transition energies, oscillator strengths, and total cross sections for the first two dipole excitations of electron bubbles in ${ }^{3} \mathrm{He}$ calculated with the FR functional at $T=0$ and $P=0 \mathrm{~atm}$.

\begin{tabular}{cccc}
\hline \hline Transition & $\begin{array}{c}\text { Transition } \\
\text { energy }(\mathrm{eV})\end{array}$ & $\begin{array}{c}\text { Oscillator } \\
\text { strength }\end{array}$ & $\begin{array}{c}\text { Total cross } \\
\text { section }\left(\mathrm{eV} \mathrm{cm}{ }^{2}\right)\end{array}$ \\
\hline $1 s-1 p$ & 0.071 & 0.972 & $1.07 \times 10^{-16}$ \\
$1 s-2 p$ & 0.326 & 0.0248 & $2.73 \times 10^{-18}$ \\
\hline \hline
\end{tabular}

approach, in conjunction with a realistic electron-helium interaction, is able to reproduce without any further assumption, the low temperature infrared spectrum of electron bubbles experimentally determined by Grimes and Adams. ${ }^{4,5}$ On the other hand, the method yields results that agree with the experimental findings of Maris and co-workers on cavitation of electron bubbles below saturation pressure, ${ }^{6,50}$ in a wide range of temperatures. ${ }^{29}$

We have shown that the analysis of infrared absorption transitions of electron bubbles constitute a stringent test for the theoretical models aiming a detailed description of the free surface of liquid helium, in particular of its surface thickness. This is at variance with electron bubble cavitation, which seems to be sensitive only to global properties of the surface, like its tension. Indeed, we have checked that the cavitation pressure for electron bubbles in liquid ${ }^{4} \mathrm{He}$ yielded by the zero-range DF is -2.07 bar, whereas the OP functional yields -2.13 bar, and OT yields -2.08 bar. These differences are far smaller than the experimental error bars, and are partly due to the small differences in the surface tensions predicted by these functionals.

There are some related problems that can be studied as a natural extension of the work carried out until the present within the DF frame; in particular, the effect on the critical cavitation pressure of quantized vortices pinned to excess electrons. ${ }^{6}$ The infrared absorption above $T \sim 2 \mathrm{~K}$ is another open problem whose quantitative description requires relaxation of the Franck-Condon principle. Although they were not detected in the experiments, ${ }^{4,5}$ it would be also interesting to check if there are other resonances in the infrared spectrum arising from the state where the helium atoms are relaxed, even though this state itself is not realized. For instance, in the case of electrons in crystals, this phenomenon, pertaining to polaron physics, is the origin of a peak in the electron optical spectrum on top of the Franck-Condon spectrum, due to the resonance of this virtual relaxed excited state with the applied radiation field. ${ }^{51}$ Both problems, presently insoluble by any microscopic approach, can be addressed within DF and time-dependent DF theories. As these are not trivial issues at all, they may be the next test grounds to assess the capabilities and limitations of the DF theory applied to liquid helium.

\section{ACKNOWLEDGMENTS}

We would like to thank F. Ancilotto, E. S. Hernández, and H. J. Maris for useful discussions. This work has been performed under Grants No. FIS2005-01414 from DGI, Spain (FEDER), and No. 2005 SGR00343 from Generalitat de Catalunya.

\section{APPENDIX}

In this appendix we work out the zero-temperature surface tension of bulk liquid ${ }^{4} \mathrm{He}$ as a function of $P$ for the zerorange DF. As we have previously stated, this is a model system, since bulk helium can only be in equilibrium with its vapor along the coexistence line, and thus for one single $P$ value at the given $T$. 


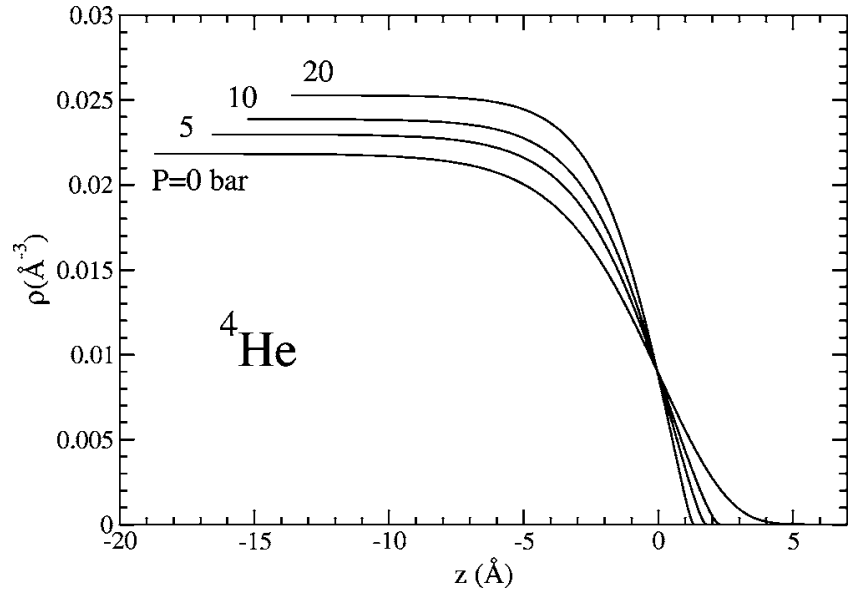

FIG. 8. Density profiles ( $⿱$ A) for the overpressurized liquid ${ }^{4} \mathrm{He}$ model system described in the Appendix, as a function of $r(\AA)$ for several values of $P$ (bar). The results have been obtained using the zero-range DF.

We closely follow the method developed in Refs. 30 and 38 . We take the planar interface perpendicular to the $z$ axis, and consequently, the liquid density is only a function of $z$. At given $P$, the equation of state determines the value of the bulk density $\rho_{b}$ and chemical potential $\mu$. Starting from Eqs. (3) and (4) taken at $T=0$, one writes the Euler-Lagrange equation

$$
\frac{d f_{v o l}}{d \rho}-\beta\left(\frac{\rho^{\prime 2}}{\rho}+2 \frac{\rho^{\prime \prime}}{\rho}\right)-2 \xi \rho^{\prime \prime}=\mu,
$$

where the prime denotes the $z$ derivative. Equation (A1) admits solutions that go to $\rho_{b}$ when $z \rightarrow-\infty$, and to zero when $z \rightarrow \infty$. For the Euler-Lagrange equation (A1), it is easy to obtain $\rho(z)$ —actually $z(\rho)$ —as indicated in Refs. 30 and 38. Density profiles corresponding to $P=0,5,10$, and 20 bar are shown in Fig. 8 as a function of $z$. Note that for each profile, the location of the $z=0$ point is arbitrary, as it stems from the structure of Eq. (A1), and that the surface region is steeper the higher the pressure is. The variation with pressure of the surface thickness is shown in Fig. 9.

To obtain the surface tension, we first define a reference system in which surface effects are not taken into account. It is a step-density system of bulk density $\rho_{b}$ filling the $z<0$ half space, whose free surface is arbitrarily located at $z=0$. Placing-also arbitrarily - the overpressurized ${ }^{4} \mathrm{He}$ density profile in such a way that $\rho(z=0)=\rho_{b} / 2$, we obtain $\sigma(P)$, subtracting the grand potential of the overpressurized system, $\omega=f(\rho)-\mu \rho$, from that of the reference system, $\omega_{b}$ $=f_{v o l}\left(\rho_{b}\right)-\mu \rho_{b}$, if $z \leqslant 0$, and 0 anywhere else, and dividing the difference by the surface of the planar interface.

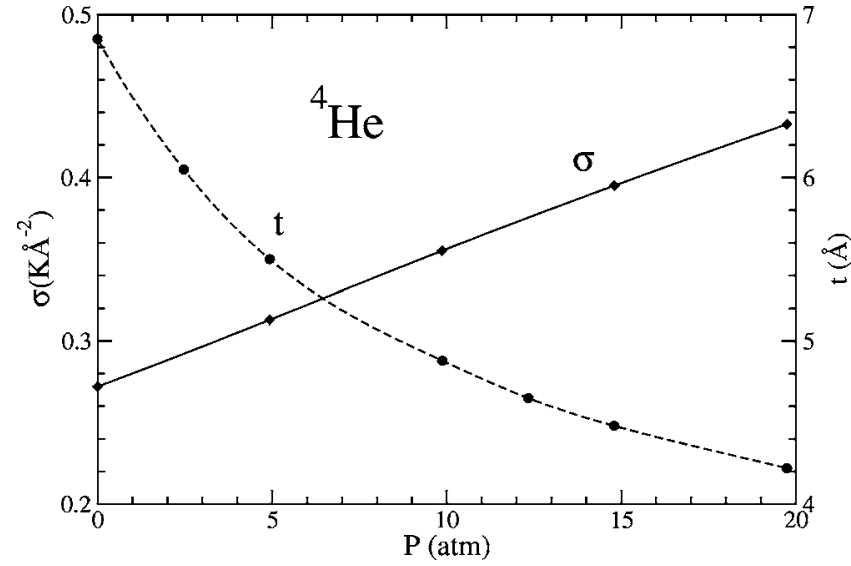

FIG. 9. Surface tension ( $\mathrm{K} \AA^{-2}$, left scale) at zero temperature as a function of $P$ (atm) for the overpressurized liquid ${ }^{4} \mathrm{He}$ model system described in the Appendix. Also shown is the surface thickness $t$ ( $\mathrm{A}$, right scale). The results have been obtained using the zero-range DF.

Defining

$$
\Delta f=f_{\text {vol }}(\rho)-f_{\text {vol }}\left(\rho_{b}\right), \quad \Delta \rho=\rho-\rho_{b},
$$

we have

$$
\begin{aligned}
\sigma(P)= & 2 \int_{-\infty}^{0}(\Delta f-\mu \Delta \rho) d z \\
& +2 \int_{0}^{\infty}\left(f_{v o l}(\rho)-\mu \rho+\beta \frac{\rho^{\prime 2}}{\rho}+\xi \rho^{\prime 2}\right) d z .
\end{aligned}
$$

Using that Eq. (A1) can be integrated to yield ${ }^{38}$

$$
\frac{d \rho}{d z}=-\left(\frac{\Delta f-\mu \Delta \rho}{\beta / \rho+\xi}\right)^{1 / 2}
$$

a close formula for the surface tension can be readily obtained:

$$
\begin{aligned}
\sigma(P)= & 2 \int_{\rho_{b} / 2}^{\rho_{b}}(\Delta f-\mu \Delta \rho)^{1 / 2}\left(\frac{\beta}{\rho}+\xi\right)^{1 / 2} d \rho \\
& +\int_{0}^{\rho_{b} / 2} \frac{f_{v o l}(\rho)-\mu \rho+\Delta f-\mu \Delta \rho}{(\Delta f-\mu \Delta \rho)^{1 / 2}}\left(\frac{\beta}{\rho}+\xi\right)^{1 / 2} d \rho .
\end{aligned}
$$

Note that the explicit knowledge of $\rho(z)$ is not needed to determine $\sigma$. This is due to the particular form of Eq. (A1). ${ }^{38}$

Figure 9 shows the surface tension $\left(\mathrm{K}^{-2}\right)$ as function of $P(\mathrm{~atm})$. It can be seen that in the displayed pressure range, $\sigma(P)$ increases almost linearly. The variation we find from 0 to $20 \mathrm{~atm}$ is similar to that found in Ref. 17 . 
${ }^{1}$ J. A. Northby and T. M. Sanders, Phys. Rev. Lett. 18, 1184 (1967).

${ }^{2}$ W. B. Fowler and D. L. Dexter, Phys. Rev. 176, 337 (1968).

${ }^{3}$ T. Miyakawa and D. L. Dexter, Phys. Rev. A 1, 513 (1970).

${ }^{4}$ C. C. Grimes and G. Adams, Phys. Rev. B 41, 6366 (1990).

${ }^{5}$ C. C. Grimes and G. Adams, Phys. Rev. B 45, 2305 (1992).

${ }^{6}$ J. Classen, C. -K. Su, M. Mohazzab, and H. J. Maris, Phys. Rev. B 57, 3000 (1998).

${ }^{7}$ D. Konstantinov and H. J. Maris, Phys. Rev. Lett. 90, 025302 (2003).

${ }^{8}$ P. Grinfeld and H. Kojima, Phys. Rev. Lett. 91, 105301 (2003).

${ }^{9}$ J. Tempere, I. F. Silvera, and J. T. Devreese, Phys. Rev. Lett. 87, 275301 (2001).

${ }^{10}$ J. Tempere, I. F. Silvera, and J. T. Devreese, Phys. Rev. B 67, 035402 (2003).

${ }^{11}$ H. J. Maris, J. Low Temp. Phys. 132, 77 (2003).

${ }^{12}$ M. Fárník, U. Henne, B. Samelin, and J. P. Toennies, Phys. Rev. Lett. 81, 3892 (1998).

${ }^{13}$ H. J. Maris and W. Guo, J. Low Temp. Phys. 137, 491 (2004).

${ }^{14}$ W. T. Sommer, Phys. Rev. Lett. 12, 271 (1964); M. A. Woolf and G. W. Rayfield, ibid. 15, 235 (1965).

${ }^{15}$ P. Roche, G. Deville, N. J. Appleyard, and F. I. B. Williams, J. Low Temp. Phys. 106, 565 (1997).

${ }^{16}$ M. Iino, M. Suzuki, and A. J. Ikushima, J. Low Temp. Phys. 61, 155 (1985).

${ }^{17}$ D. Amit and E. P. Gross, Phys. Rev. 145, 130 (1966).

${ }^{18} \mathrm{R}$. Evans, in Liquids at Interfaces, edited by J. Charvolin, J. F. Joanny, and J. Zinn-Justin (Elsevier, Amsterdam, 1989), p. 1.

${ }^{19}$ F. Ancilotto and F. Toigo, Phys. Rev. B 50, 12820 (1994).

${ }^{20}$ J. Dupont-Roc, M. Himbert, N. Pavloff, and J. Treiner, J. Low Temp. Phys. 81, 31 (1990).

${ }^{21}$ N. R. Kestner, J. Jortner, M. H. Cohen, and S. A. Rice, Phys. Rev. 140, A56 (1965).

${ }^{22}$ J. Eloranta and V. A. Apkarian, J. Chem. Phys. 117, 10139 (2002).

${ }^{23}$ L. Giacomazzi, F. Toigo, and F. Ancilotto, Phys. Rev. B 67, 104501 (2003).

${ }^{24}$ L. Lehtovaara, T. Kiljunen, and J. Eloranta, J. Comput. Phys. 194, 78 (2004).

${ }^{25}$ F. Dalfovo, A. Lastri, L. Pricaupenko, S. Stringari, and J. Treiner, Phys. Rev. B 52, 1193 (1995).

${ }^{26}$ Q. Xiong and H. J. Maris, J. Low Temp. Phys. 77, 347 (1989).

${ }^{27}$ M. Barranco, M. Guilleumas, M. Pi, and D. M. Jezek, in Microscopic Approaches to Quantum Liquids in Confined Geometries, edited by E. Krotscheck and J. Navarro (World Scientific, Singapore, 2002), p. 319.

${ }^{28}$ S. Balibar, J. Low Temp. Phys. 129, 363 (2002).

${ }^{29}$ M. Pi, M. Barranco, R. Mayol, and V. Grau, J. Low Temp. Phys. 139, 397 (2005).
${ }^{30}$ A. Guirao, M. Centelles, M. Barranco, M. Pi, A. Polls, and X. Viñas, J. Phys.: Condens. Matter 4, 667 (1992).

${ }^{31}$ E. Cheng, M. W. Cole, and M. H. Cohen, Phys. Rev. B 50, 1136 (1994); 50, 16134(E) (1994).

${ }^{32}$ M. Rosenblit and J. Jortner, Phys. Rev. B 52, 17461 (1995).

${ }^{33}$ W. H. Press, S. A. Teulosky, W. T. Vetterling, and B. P. Flannery, Numerical Recipes in Fortran 77: The Art of Scientific Computing (Cambridge University Press, Cambridge, UK, 1999).

${ }^{34}$ S. Stringari and J. Treiner, J. Chem. Phys. 87, 5021 (1987); Phys. Rev. B 36, 8369 (1987).

${ }^{35}$ J. Harms, J. P. Toennies, and F. Dalfovo, Phys. Rev. B 58, 3341 (1998).

${ }^{36}$ K. Penanen, M. Fukuto, R. K. Heilmann, I. F. Silvera, and P. S. Pershan, Phys. Rev. B 62, 9621 (2000).

${ }^{37}$ J. Harms, J. P. Toennies, M. Barranco, and M. Pi, Phys. Rev. B 63, 184513 (2001).

${ }^{38}$ M. Barranco, M. Pi, A. Polls, and X. Viñas, J. Low Temp. Phys. 80, 77 (1990).

${ }^{39}$ D. O. Edwards and W. F. Saam, Prog. Low Temp. Phys. 7A, 285 (1978).

${ }^{40}$ D. V. Osborne, J. Phys.: Condens. Matter 1, 289 (1989).

${ }^{41}$ J. M. Marín, J. Boronat, and J. Casulleras, Phys. Rev. B 71, 144518 (2005).

${ }^{42}$ This is achieved by making $T$ dependent the characteristic distance $h$ at which the Lennard-Jones potential of OP is screened (Ref. 20), in such a way that at given $T$, the volume integral of the screened potential equals the parameter $b(T)$ in Eq. (4), and taking the parameter $c$ of the OP (Ref. 20) equal to the $c(T)$ parameter in Eq. (4). These changes are complemented with the inclusion in the $T$-dependent OP functional of the free energy of the noninteracting Bose gas, as done in Ref. 30.

${ }^{43}$ F. Ancilotto, F. Faccin, and F. Toigo, Phys. Rev. B 62, 17035 (2000).

${ }^{44}$ To avoid overcrowding this figure, we have not represented here the results obtained in Ref. 4 using their spherical-square-well model, nor those obtained by the same authors using the approach of Ref. 3. They can be seen in Fig. 2 of Ref. 4.

${ }^{45}$ J. Jortner, N. R. Kestner, S. A. Rice, and M. H. Cohen, J. Chem. Phys. 43, 2614 (1965).

${ }^{46}$ B. E. Springett, Phys. Rev. 155, 139 (1967).

${ }^{47}$ R. M. Ostermeier, Phys. Rev. A 8, 514 (1973).

${ }^{48}$ T. Ellis, P. V. E. McClintock, and R. M. Bowley, J. Phys. C 16, L485 (1983).

${ }^{49}$ M. Inno, M. Suzuki, A. J. Ikushima, and Y. Okuda, J. Low Temp. Phys. 59, 291 (1985).

${ }^{50}$ C. -K. Su, C. E. Cramer, and H. J. Maris, J. Low Temp. Phys. 113, 479 (1998).

${ }^{51}$ J. Devreese, J. De Sitter, and M. Goovaerts, Phys. Rev. B 5, 2367 (1972). 